\title{
The scheme of dosing additives to fuel oil and evaluation of the effectiveness of its implementation at the enterprises of the fuel and energy complex
}

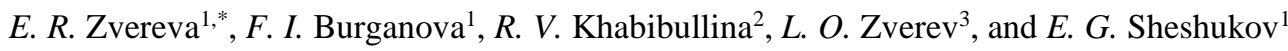 \\ ${ }^{1}$ Kazan State Power Engineering University, Kazan, Russia \\ ${ }^{2}$ LLC Engineering Center "Enegoprogress", Kazan, Russia \\ ${ }^{3}$ St. Petersburg State University of Industrial Technology and Design, St. Petersburg, Russia
}

\begin{abstract}
Additives are actively used to improve the quality of liquid fuels. Effective mixing of the additive with fuel with high reliability and efficiency of the boiler is ensured by the choice of technological dosing scheme liquid additive which will allow to organize automatically preparation of the additive, adding it to the oil and stirring.
\end{abstract}

\section{Introduction}

The peculiarity of the modern oil refining industry is the tendency to deepen oil refining, which is explained by the limited reserves and the tightening of environmental requirements for oil products.

However, as oil refining deepens, the share of straight-run fuel oil in boiler fuels decreases, and the share of tar and heavy cracked residues increases, that is, the quality of fuel oil deteriorates. Reducing the quality of fuel oil for the consumer means a deterioration of his physical stability and a decrease in the efficiency of burning [1-4].

\section{Additive dosing methods to fuel oil}

Currently, the most used and common way to combat sediment, improve the quality of liquid fuels is the use of various additives. The use of additives depends little on the design and mode of operation of the boiler; their input into fuel oil can be organized outside the power plant [5-8].

Additives can exist in various aggregative states. To prevent severe corrosion and clogging of the boiler tubes, the following additives may be used: magnesite, dolomite, lime, liquid additives and ammonia gas. Solid additives - magnesite, dolomite and lime, introduced into the gas ducts of the boiler in the form of finely ground powder, did not find wide application. Additional large capital costs are required for the grinding of these materials, and the operation of the equipment is considerably complicated. Liquid additives are most common.

Additives are introduced into the fuel in various ways, the choice of which depends on the amount of the additive, its physico-chemical characteristics and purpose, as well as features of application. In all cases, it is required to ensure effective mixing of the additive with the fuel at the lowest energy and labor costs [9-11].

The preparation of a liquid concentrate is necessary for the convergence of the physicochemical characteristics of the fuel and the additive, which guarantees good mixing.

When fuel is shipped, the additive in the calculated amount can be poured into an empty or partially filled tank. With further fuel filling occurs a sufficiently effective mixing occurs, which continues during transportation. However, we must bear in mind that the additives are heavier than fuel and sink to the bottom of the tank. From this point of view, it is desirable that the difference between the density of the fuel and the additive be minimized.

Unloading additives from small containers and its preparation is often combined into one process. The additive can be prepared in tanks 8 (Figure 1) equipped with a temperature sensor 4, which determines the temperature of the additive.

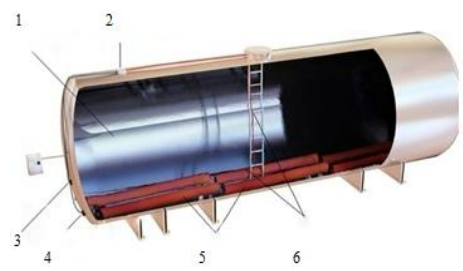

Fig. 1. Storage tank additives: 1-heated fluid; 2-junction box; 3-level sensor; 4-temperature sensor; 5-submersible fiberglass heaters; 6-heat and oil resistant wire; heat control automation.

A change in the temperature environment leads to a change in the pressure of the fluid inside the sensor, which in turn, through a system of levers, closes or opens the contacts of the device. Heating of the additive in the tank is allowed to a temperature not lower than 20

Corresponding author: 6elvira6@list.ru 
and not higher than 50 . There is also a circulation loop to prevent the additive from freezing. Circulating mixing is carried out by the forced circulation of the liquid, so that turbulence arises that promotes mass transfer.

Heating of the additive can be carried out on the basis of submersible fiberglass heaters 14 . The heater is a fiberglass pipe, carbon fiber material is used as a heating element and is placed inside the pipe wall.

Heaters are installed in the tank and are in contact directly with the heated medium, ensuring maximum efficiency of electric heating.

Active mixing of the heated medium occurs due to the combined effect of two physical processes:

- the effect of free (natural) convection, in which the lower layers of the substance heat up and float up, and the top layers cool down and sink down;

- Venturi effect, in which the flow rate of a substance increases when it gets inside the heater-pipe, and additional heating of the substance from the inner walls of the heater greatly enhances this effect.

\section{Results and discussion}

The additive storage tank has a level 3 sensor with which the software logic controller 1 monitors the additive content in the tank. Level control is based on converting the change in electrical resistance between the tank wall and the sensor electrode into an electrical relay signal. When immersed in the controlled environment of the electrode sensor, this resistance decreases and the corresponding channel relay operates, the LED lights up. If there is no medium, the resistance increases, the relay is de-energized, the LED goes out. In the case of filling the tank, the exhaust valve 2 opens and excess additive is removed.

After exiting the storage tank, the additive passes through the filter 5 , which is intended for cleaning from mechanical impurities, then the purified additive is pumped by the metering pump 7. The flow meter 9 is installed on the additive supply line; according to its parameters, the controller controls the consumption of the additive before it is fed to the fuel oil pipeline. A check valve 10 is installed to prevent the back flow of the additive in the supply line. A flow meter 9 is installed on the main fuel oil pipeline. Of the total volume of the main fuel oil flow, $2 \%$ is taken for mixing with the additive. This stream is pumped by the pump 13. Before adding an additive to the fuel oil, the latter in turn passes through the steam heater 11 , which is designed to preheat the fuel oil and thus to prevent it from freezing, as well as to provide the necessary viscosity values.

After passing through the steam heater, the temperature of the fuel oil is measured and the additive is added to the fuel oil pipeline. Mixing of fuel oil and additives is carried out by means of a static mixer 12 .

After passing through the steam heater, the temperature of the fuel oil is measured and the additive is added to the fuel oil pipeline. Mixing of fuel oil and additives is carried out by means of a static mixer 12 .

Static mixing refers to mixing without moving mechanical devices. Products are mixed only due to the energy of the stream with the participation of fixedly mounted mixing elements that contribute to the continuous separation and redistribution of the total flow over the cross section of the mixing channel.

Next, a mixture of fuel oil and additives is fed into the main fuel oil pipeline. The oil in which the additive is introduced must be heated to a temperature of 50-70. The scheme of dosing additives in fuel oil is presented in Figure 2 [12].

The developed technological scheme for dosing a liquid additive will allow automatic preparation of the additive, adding it to the fuel oil, thereby ensuring high reliability and efficiency of the boiler.

Instead of heaters to reduce viscosity and prevent freezing of the additive, you can use a mixing device.

The mixing process is used to obtain homogeneous or heterogeneous mixtures. With mixing, a uniform distribution of the phases in the whole volume and their close interaction is achieved.

For carrying out the mixing process using the following main methods:

1) mechanical, in which the mixing is carried out by various rotating devices;

2) bubbling, carried out by passing a gas through a layer of liquid;

3) hydraulic, carried out by mixing the streams during their joint movement in the channel or when pumping the mixed media by the pump.

The use of mixers (paddle, propeller, turbine, etc.) makes it possible to most effectively solve the problems of averaging the characteristics of products in tanks and preventing bottom sediments [13-15].

Propeller mixers are used for intensive mixing of liquids, stirring up sediments containing up to $10 \%$ of the solid phase with particle sizes up to $0.15 \mathrm{~mm}$, as well as for preparing suspensions and emulsions.

The mixers are mounted on the hatch of the first belt of the tank. The agitator shaft is parallel to the bottom of the tank. Under the influence of the submerged jet from the propeller, the product in the tank is intensively mixed, the available bottom sediments are eroded. When the output shaft of the agitator with the propeller is rotated at an angle of up to $30^{\circ}$ from the neutral position, the product is given a rotational motion in the tank, which allows the characteristics of the product to be aligned throughout the tank volume. To obtain uniformity of product characteristics in tanks of large volumes (5000 $\mathrm{m} 3$ and above), the direction of rotation of the product in the tank can be reversed by turning the output shaft of the agitator in the ball bearing.

The use of agitators gives the following advantages:

- energy consumption with stirring is significantly lower than with stirring by the circulation of the product from the tank to the tank with the help of pumps; 


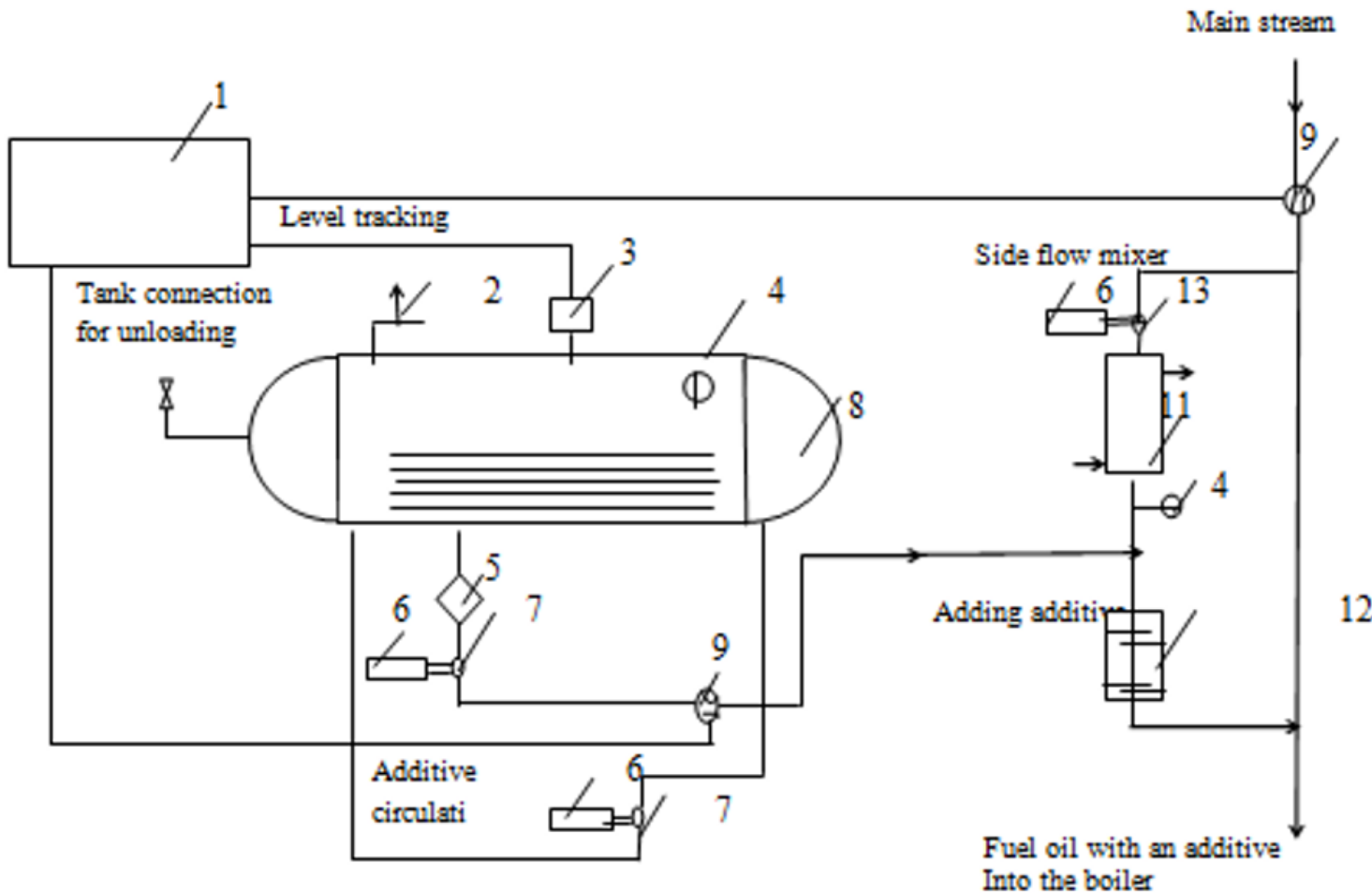

Fig. 2. Scheme of dosing additives in fuel oil: 1-programming logic controller; 2- exhaust valve; 3-level sensor; 4-temperature sensor; 5-filter; 6-generator; 7-additive dosing pump and circulation pump; 8-capacity storage; 9-flow meter; 10-check valve; 11- steam heater; 12- static mixer; 13-fuel oil metering pump.

- bottom sediments in the tanks are practically absent, the cost of cleaning the tanks during operation is minimal;

- there is no moistening or oxidation of the product as when mixing by blowing the tanks with air.

The effectiveness of the mixing device characterizes the quality of the mixing process. The quality of mixing depends not only on the design of the mixing device and apparatus, but also on the amount of energy introduced into the mixed liquid. The effectiveness of mixing is also the basis for evaluating the operation of the same apparatus (to select the optimal mode of operation of the apparatus and its optimal dimensions) [16-18].

The intensity of mixing is determined by the time to achieve a given technological result or the number of revolutions of the stirrer for a fixed duration of the process. The higher the intensity of mixing, the less time is required to achieve a given mixing effect. Intensification of mixing processes leads to a decrease in the size of the designed equipment and an increase in the current productivity.

The defining equation for the Reynolds criterion:

$$
\operatorname{Re}=\frac{n d^{2} \rho}{\mu}
$$

where $\mathrm{n}$ - is the number of revolutions of the stirrer, $\mathrm{rpm} ; \mathrm{d}-$ is the diameter of the stirrer, $\mathrm{m} ; \rho$-density (at $\left.120^{\circ} \mathrm{C}\right) ; \mu$-dynamic coefficient of viscosity, sPz.

The net power $\mathrm{N}$ reported by the fluid is expressed in terms of the power criterion $\mathrm{K}_{\mathrm{N}}$ or the modified Euler criterion:

$$
E u_{\mathcal{M}}=\frac{N}{\rho \cdot d_{\mathcal{M}}^{5} \cdot n^{3}}=K_{N}
$$

or

$$
N=K_{N} \cdot \rho \cdot d_{\mathcal{M}}^{5} \cdot n^{3}
$$

A propeller mixer with three blades with reflective walls at $\mathrm{d}_{\mathrm{M}}=0.5 \mathrm{~m}$ has $\mathrm{N}=2.038 \mathrm{~kW}$.

For the introduction of the dosing complex additives for fuel oil with propeller mixers, it is necessary to assess its economic efficiency. The information base for calculating the effectiveness of investments is the flow of payments. The flow of payments is based on the indicators of "net income" and investment expenses. The net income $\mathrm{D}$ is understood as the total income received in each time interval $t$ minus the costs associated with its receipt. Payments include all direct and indirect costs (fuel, labor, materials, etc., as well as taxes), depreciation deductions are not included in these expenses, because these funds remain at the enterprise for compensation of depreciation of fixed assets and for expanded reproduction. The cost of implementing the equipment of the proposed scheme for dosing a liquid fuel oil additive is 1770,220 rubles.

During the calculations, social and other noneconomic effects, a risk factor and inflation were not taken into account. The tax rate was taken $24 \%$. Savings from the introduction of the dosing complex, Rt amounted to 21,289.80 thousand rubles / year, Net income, Dt, 842.34 thousand rubles / year. The index of 
profitability, ID 3.44. Payback period, months 10. The use of such a methodical approach in calculations allows one to draw fundamental conclusions about the effectiveness of the introduction of fuel oil additive within acceptable limits, taking into account the following factors: cost of the dosing device and additives, operating factors, characteristics of fuel oil, repair costs, specific fuel consumption, fuel losses at start-up boilers and regional features. The index of profitability of capital costs varies from 4.78 and higher depending on the price of fuel oil and the rate of discount, therefore, with increasing prices for fuel oil, the effectiveness of the use of additives increases [19$20]$.

\section{References}

[1] A.M. Danilov, Application of Fuel Additives (Khimizdat, St. Peterburg) 368 (2010)

[2] V.M. Kapustin, Petroleum-Based and Alternative Fuels with Additives (KolosS, Moscow) 232 (2008)

[3] E.R. Zvereva, O.S. Zueva, R.V. Khabibullina, et al Chemistry and technology of fuels and oils 5(597), 15-19 (2016)

[4] E.R. Zvereva, O.S. Zueva, R.V. Khabibullina, A.O. Makarova, Journal of Engineering and Applied Sciences 11, 2950-2954 (2017)

[5] E.R. Zvereva, R.V. Khabibullina, A.O. Makarova, G.R. Akhmetvalieva, F.I. Burganova, D.V. Ermolaev, O.S. Zueva, Petrochemistry 59, 1 98-103 (2019)

[6] E.V. Shamsutdinov, O.S. Zueva, Nanomaterials and Nanotechnologies in Power Industry, (KGEU, Kazan, 2014) 400

[7] O.S. Zueva, Y.K. Mongush, A.O. Makarova, Solid State Phenomena 284, 713-718 (2018)

[8] V.G. Kulichikhin, A.V. Semakov, V.V. Karbushev, N.A. Plate, P.J. Picken, High molecular weight compounds 51(11), 2044 (2019)

[9] A.Ya. Malkin, V.G. Kulichikhin, Advances in chemistry 84(8), 803 (2015)

[10] O.S. Zueva, Yu.N. Osin, V.V. Salnikov, Yu.F. Zuev, Basic research . 11-5, 1021 (2014)

[11] A.A. Pykhtin, P.V. Surikov, L.B. Kandyrin, V.N. Kuleznev, Vestnik MITKHT(Moscow) 8(4), 113 (2013)

[12] E.R. Zvereva, I.A. Mutugullina, R.V. Zinnatullin, Patent Ros.Federatsiya The system for the preparation of fuel oil for combustion. 2531299; declare 04/01/2013, publ .: 10/20/2014.Bul 29, 11

[13] E.R. Zvereva, G.R. Mingaleeva, R.V. Khabibullina, G.R. Akhmetvalieva, Petrochemistry 1, 73-75 (2016)

[14] E.R. Zvereva, O.S. Zueva, R.V. Khabibullina, Mater. Sci. Forum 870, 666-670 (2016)

[15] E.R. Zvereva, O.S. Zueva, R.V. Khabibullina, Z.F. Khatmullina, E.S. Dremecheva, News of higher educational institutions. Energy problems 1-2, 28-36 (2016)
[16] E.R. Zvereva, R.V. Khabibullina, G.R. Akhmetvalieva, A.O. Makarova, O.S. Zueva, Advances in Engineering Research 133, 914-920 (2017)

[17] E.R. Zvereva, R.V. Khabibullina, O.S. Zueva, Solid State Phenomena 265, 374-378 (2017)

[18] E.R. Zvereva, T.M. Farakhov, Energy-saving technologies and apparatus of thermal power plants when working on fuel oil Heat engineer (Moscow) 181 (2012)

[19] E.R. Zvereva, A.V. Dmitriev, M.F. Shageev, G.R. Akhmetvaliyeva, Thermal Engineering 8, 50-56 (2017)

[20] E.R. Zvereva, M.F. Shageev, A.V. Dmitriev, others Vestnik of KGEU (KGEU, Kazan) 10 . 1(37), 6473 (2018) 